\title{
"Non-lieu" ou espace de l'entre-deux ? La place des saisonniers dans les stations de sports d'hiver haut de gamme des Alpes françaises du nord
}

\section{Lise Piquerey}

\section{(2) OpenEdition}

Journals

Édition électronique

URL : http://journals.openedition.org/cdg/510

DOI : $10.4000 /$ cdg. 510

ISSN : 2107-7266

Éditeur

UMR 245 - CESSMA

\section{Référence électronique}

Lise Piquerey, «"Non-lieu" ou espace de l'entre-deux ? La place des saisonniers dans les stations de sports d'hiver haut de gamme des Alpes françaises du nord », Carnets de géographes [En ligne],

7 | 2014, mis en ligne le 01 décembre 2014, consulté le 30 avril 2019. URL : http:// journals.openedition.org/cdg/510 ; DOI : 10.4000/cdg.510

\section{(c) (†) $\odot$}

La revue Carnets de géographes est mise à disposition selon les termes de la Licence Creative Commons Attribution - Pas d'Utilisation Commerciale - Pas de Modification 4.0 International. 


\title{
"Non-lieu" ou espace de l'entre-deux? \\ La place des saisonniers dans les stations de sports d'hiver haut de gamme des Alpes françaises du nord
}

LISE PIQUEREY

Doctorante en géographie Laboratoire EDYTEM CNRS UMR 5204 - Université de Savoie Lise.piquerey@univ-savoie.fr

\section{Résumé}

De par leur forte vulnérabilité à la saisonnalité, les lieux touristiques semblent être un cas particulièrement adapté pour mener une réflexion sur les notions de non-lieu et d'espace de l'entre deux. Dépassant le cadre touristique, cet article vise à questionner la considération et la perception des lieux de vie des travailleurs saisonniers au sein des stations de sports d'hiver haut de gamme. Quelle place occupent ces espaces, souvent ignorés, évités voire relégués, dans l'agencement de ces stations ? En quoi peuvent-ils être considérés comme des espaces de l'entre deux, relevant d'un processus constitutif de nouvelles spatialités?

\begin{abstract}
By their high vulnerability to seasonal, tourist places seem to be particularly suitable for a case to reflect on the concept of non-place and space in between. Exceeding the tourism context, this article aims to question the character and perception of the lives of seasonal workers in the winter sports resorts upscale. What is the role that these areas often ignored, avoided or relegated involved in the arrangement of these stations? How can they be considered as spaces between the two, within a constitutive process new spatiality?
\end{abstract}




\section{Introduction}

Le 15 janvier 2013, l'Humanité titrait : "Drame de La Clusaz : la mort attendait les jeunes saisonniers dans l'ombre "; plus qu'un simple fait divers hivernal, cet événement (décès des deux saisonniers dans l'incendie de leur caravane) remet en lumière une problématique centrale au sein des stations de sports d'hiver : où loger les saisonniers ? Ces travailleurs, décrits par leurs employeurs comme des personnes mobiles, jeunes et aux comportements parfois excessifs, sont les petites mains des grandes destinations touristiques française. Cet article vise à questionner leur place dans les stations de sports d'hiver haut de gamme en Savoie et en Haute-Savoie (Megève, Courchevel, Val d'Isère et Val Thorens). Le choix de ces quatre stations d'étude relève d'une grille d'échantillonnage permettant d'identifier une sélectivité de la clientèle à travers les types d'hébergements, les prix des forfaits de remontées mécaniques, les différentes boutiques de marque haut de gamme et les événements proposés; elle s'appuie sur une élitisation, à travers une montée en gamme des stations de sports d'hiver. Elle se matérialise notamment par le développement des infrastructures qui leur sont propres et adaptées, comme l'ouverture d'un nouvel hôtel cinq étoiles en décembre 2013 respectivement à Courchevel 1850 et à Val Thorens. Si dans le cas de Megève, la sélectivité de la clientèle fut placée comme un principe de création du lieu touristique (Socquet, 1980 ; MIT, 2005), dans le cas de Courchevel (Leprêtre, 1986), Val d'Isère et plus récemment de Val Thorens, elle apparaît plus tardivement, à partir des années 1990. Cette montée en gamme induit également une augmentation des prix du foncier, qui se traduit par des difficultés à se loger à la fois pour les populations permanentes, dont certaines exercent une activité touristique saisonnière, mais également pour les travailleurs saisonniers, dont le nombre augmente de façon corrélative à cette montée en gamme des infrastructures touristiques. Ainsi, comme dans le cas de Courchevel, se produit une externalisation des lieux de vie des populations anciennement permanentes et des populations saisonnières dans les fonds de vallée. Si les autorités locales ont pris conscience du départ de ces populations, et cherchent à créer des nouveaux projets immobiliers et à instaurer des facilités d'accession à la propriété pour les individus ayant les revenus les plus faibles et souhaitant résider à l'année en station, il semble que les questions relatives aux logements des saisonniers soient considérées comme une problématique qui doit être gérée avant tout par les acteurs privés (les employeurs). Les saisonniers représentent 25000 emplois (INSEE, tableaux statistiques, 2010) sur la totalité du territoire de Tarentaise et de l'Espace Mont Blanc. La capacité des stations de sports d'hiver (et encore plus celles de l'échantillon fortement productrices d'emplois) à loger les saisonniers est donc un des défis principaux qu'elles doivent relever.

Ainsi, il semble que ce soit au sein des stations haut de gamme, marquées par l'exclusivité, que les fractures sociales et les disparités spatiales entre les populations les plus aisées (clientèle, activités touristiques valorisées et valorisables) et celles qui le sont moins (les 
travailleurs saisonniers) soient les plus frappantes. Dans cet article, il sera uniquement question des saisonniers qui n'appartiennent pas aux populations permanentes, de ces lieux touristiques. Cette première précision peut sembler arbitraire, mais est pourtant induite par l'objet de cette réflexion, puisque les populations permanentes qui exercent au sein de leur lieu de vie quotidien ne sont pas productrices de nouveaux lieux à travers une socialisation de lieu perçu jusqu'alors comme des lieux de passage, faiblement considérés au sein de l'espace social. Qu'ils soient appelés " maisons de saisonniers ", "foyers de travailleurs » ou " campements ", les lieux dans lesquels résident les saisonniers ont la particularité d'être des non-lieux, c'est-à-dire des espaces de transit, créés par un acteur ou un groupe d'acteurs dans l'optique de répondre à de nouveaux besoins, selon la définition de M. Augé (1992). Ces non-lieux s'inscrivent dans un réseau, car ils sont reliés à d'autres qui peuvent aussi être temporaires, mais qui sont cependant institutionnalisés, fortement visibles, comme dans le cas des hôtels cinq étoiles. La temporalité d'occupation d'un lieu est un des fondements des espaces touristiques, eux aussi appelés à être de passage du fait d'une pratique touristique marquée par la saisonnalité, la durée d'ouverture des remontées mécaniques ou encore le temps de location d'un appartement.

Le statut de travailleurs saisonniers se définit par l'exercice d'un emploi au sein d'un établissement dont l'activité n'excède pas neuf mois par an ; il regroupe une multiplicité de situations professionnelles, de stratégies de mobilités (Uriely, 2001), révélatrices de grandes disparités au sein de ce groupe de populations : le point commun entre une femme de chambre dans un centre de vacances, un moniteur de ski et un chef étoilé de palace est que tous trois possèdent un contrat de travail saisonnier. S'attachant plus à étudier les conditions de vie des saisonniers extérieurs au sein des stations de sports d'hiver haut de gamme qu'à questionner le bien fondé de leur statut, nous proposerons des pistes de réflexions pour comprendre en quoi les saisonniers, présents temporairement dans les stations de sports d'hiver haut de gamme, sont des créateurs de sociabilité(s) ponctuelle(s), transposant des lieux de " passage » en lieux de vie.

Les places (Lussault, 2009) sur lesquelles résident les saisonniers ne sont-elles pas des espaces de l'entre-deux, pratiqués seulement par les saisonniers, au sein des stations de sports d'hiver haut de gamme en Savoie et en Haute-Savoie ? Après avoir présenté la méthodologie mise en œuvre dans l'analyse des stratégies résidentielles des saisonniers, deux types de lieu seront analysés : les maisons mises à disposition des employés par les groupes hôteliers, et les espaces auto-appropriés par les saisonniers qui logent dans des camions ou des caravanes pendant les saisons touristiques hivernales, soit par choix, soit par obligation. Ces réflexions permettront de formaliser le rôle des lieux de vie des saisonniers dans la transposition de non-lieu en espaces de l'entre deux au sein des stations de sports d'hiver haut de gamme. 


\section{Visibilité, ordinarité, invisibilité : comment appréhender le terrain des saisonniers ?}

Les méthodes utilisées pour appréhender la place des saisonniers dans les stations de sports d'hiver haut de gamme sont liées à la difficulté inhérente au traitement de ce sujet. Si ces derniers sont visibles dans leur activité professionnelle, leurs lieux de vie et de socialisation semblent eux plus délicats à observer. Trois "postures " méthodologiques ont ainsi été développées dans le travail de terrain.

Tout d'abord, la méthode la plus simple est de se rendre visible. Elle correspond aux entretiens menés, selon une grille de questions préétablie avec les acteurs du système touristique : les gérants d'infrastructures touristiques comme les directeurs d'hôtels de luxe, les travailleurs saisonniers, les élus et directeurs des services communaux et les responsables et les cadres des offices de tourisme. Au fil des observations de terrain, des entretiens moins formels sont réalisés avec des employés saisonniers, toujours en se présentant comme doctorante en Géographie.

La seconde méthode utilisée est l'observation des espaces d'étude à travers la collecte d'informations avec des photographies, des essais de cartographie à main levée et des notes, qui forment ainsi un corpus de relevés de terrain. Ils mettent en lumière les marqueurs qui relèvent des stratégies de sélection et de mise à distance des populations présentes sur les stations de sports d'hiver haut de gamme.

La dernière méthode utilisée est empruntée à l'anthropologie et à la sociologie (Pinçon et Pinçon-Charlot, 2005 ; Bouillon, 2009). Durant une saison hivernale, une méthode participante est utilisée en essayant de s'intégrer au " monde des saisonniers ", en se faisant passer pour une jeune adulte en recherche d'un travail saisonnier, une situation des plus " ordinaires ». Cette intégration, débutée lors d'un forum de travail saisonnier, s'est traduite par des entretiens auprès d'employeurs et de recruteurs pour les groupes hôteliers, par le dépôt de curriculum vitae et la recherche d'un logement pour un possible poste ; cette méthode semble fondamentale pour comprendre les stratégies résidentielles et les choix de vie des saisonniers. Cette première immersion a mis en exergue l'importance du logement dans la recherche d'emploi. Si la majorité des recruteurs avance le fait que le poste s'accompagne d'un hébergement, peu de précisions sont apportées quant à la qualité de ce dernier ; des relevés de terrain ont permis d'identifier des maisons de saisonniers (appartenant à des groupes hôteliers) réputées en mauvaise état, mais qui sont présentées lors des entretiens comme des logements décents et corrects. Le simple fait qu'un poste soit logé apparaît comme une faveur dans la bouche de certains recruteurs et à la fois comme une condition non négociable pour certains demandeurs d'emploi. La seule information dont disposent les futurs employés sur les conditions de logement lors de l'entretien est qu'ils seront possiblement logés par deux, sans aucune précision sur le nombre de chambres ou la superficie de l'appartement ou de la pièce qu'ils devront partager. 
Si les deux premières méthodes employées sont plutôt communes dans la recherche en géographie, la troisième soulève plus de questions sur le rôle et la place du chercheur à mener un tel exercice. Elle interroge sa position : un chercheur doit-il vivre ou avoir vécu une situation pour pouvoir l'analyser avec objectivité ? A cette question, aucune réponse évidente ne peut émerger. En effet, l'observation participante remet en question la capacité du chercheur à objectiver scientifiquement les faits; de plus, elle est fonction de ses caractéristiques personnelles (sexe, âge, manière d'être et de faire). Cependant, elle semble également être un bon outil pour comprendre le fonctionnement d'un groupe de populations dont les pratiques socio-spatiales sont encore fréquemment méconnues.

\section{Du lieu créé au lieu approprié : les « maisons de saisonniers »}

Ce qui est ici nommé maison de saisonniers correspond à des habitations construites ou achetées par les employeurs, notamment les groupes hôteliers, pour loger les saisonniers pendant les deux périodes touristiques principales, l'hiver et l'été. Elles sont dans un état au premier abord correct ; les employés louent fréquemment une chambre avec les sanitaires collectifs à l'extérieur. Le standing des logements tranche cependant avec la qualité des lieux dans lesquels les logés travaillent, lorsqu'il s'agit d'hôtellerie haut de gamme (figure 1 et 2).

Figure 1: Maisons de saisonniers du groupe hôtelier Sibuet.

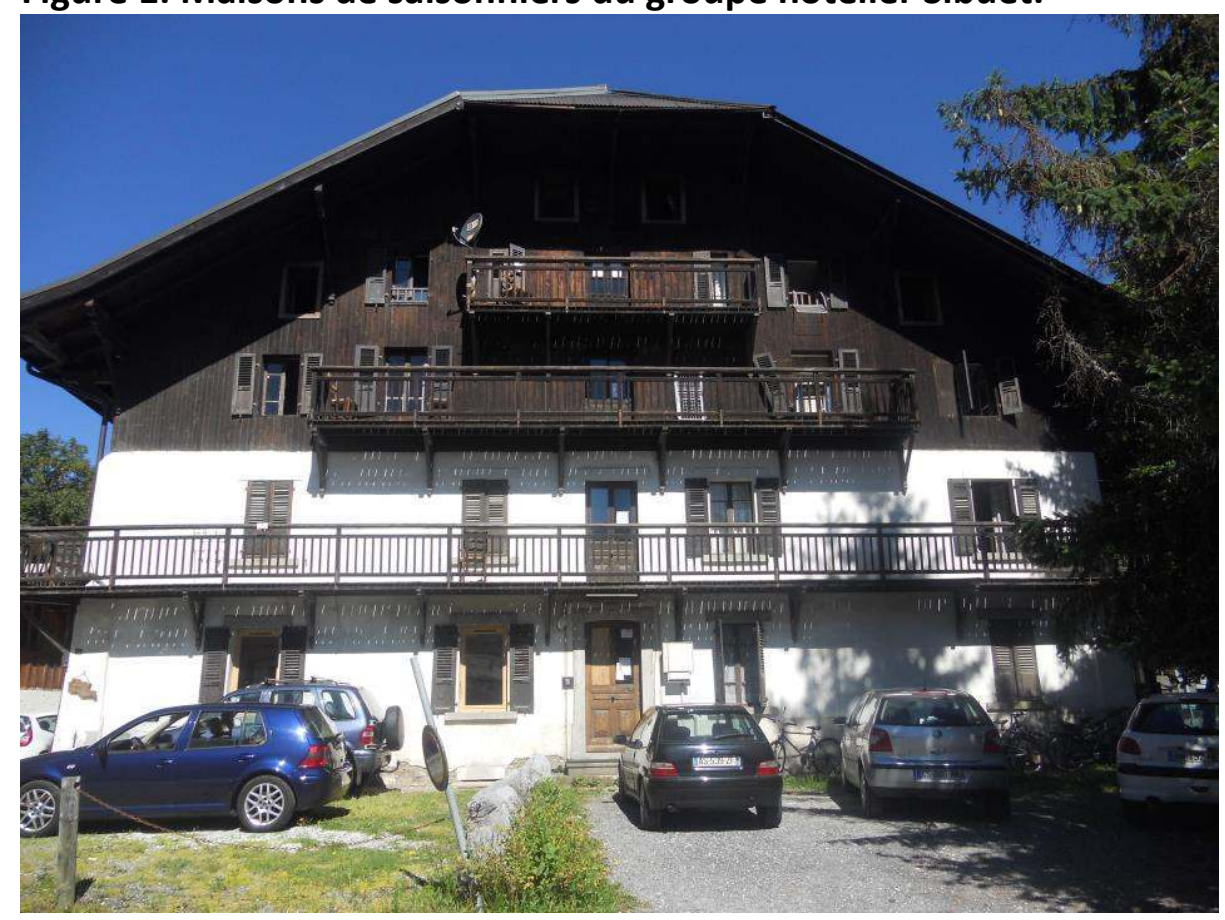

Cliché : L. Piquerey, août 2013.

Datant de 1924, louée aux employés saisonniers du groupe hôtelier haut de gamme Sibuet, propriétaire des lieux, Megève. Elle est composée de 18 chambres, avec les sanitaires et la cuisine en commun. 
Figure 2 : Entrée de l'hôtel cinq étoiles Les Fermes de Marie, groupe Sibuet, Megève.

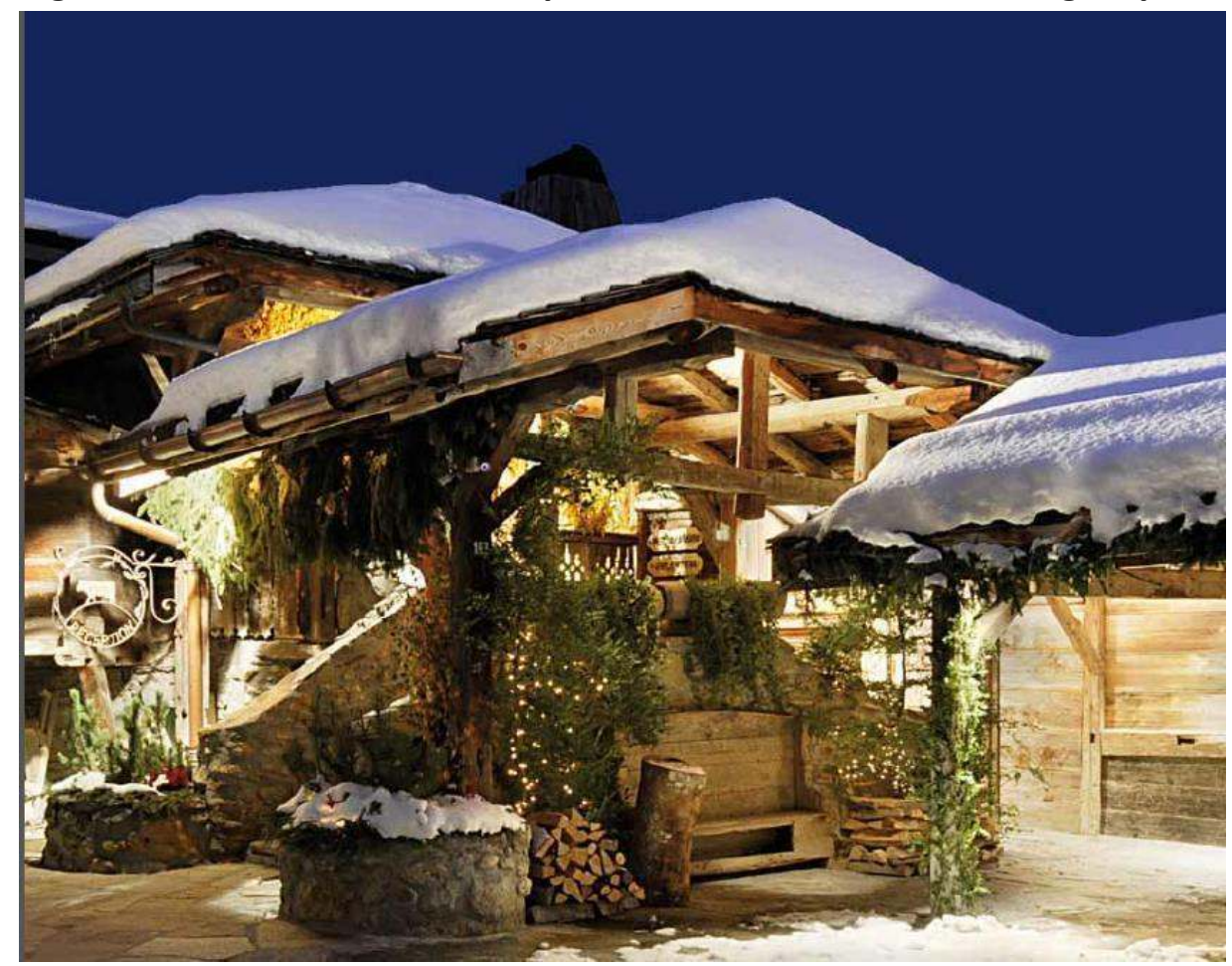

Cliché : Brochure commerciale Les Fermes de Marie, Groupe Sibuet, 2013

Ces maisons se localisent à une distance-temps d'environ quelques dizaines de minutes à pied, ou à côté du lieu de travail. Que les maisons soient visibles ou non depuis ce dernier, elles sont très peu individualisées dans le paysage. Obtenir des informations sur leur localisation se révèle assez difficile, car les employeurs restent très discrets sur les conditions d'hébergement de leurs employés. Les seules indications visibles permettant de repérer les maisons de saisonniers dans les stations sont celles laissées sur les boîtes aux lettres, précisant que le courrier doit être déposé à I'hôtel. Lorsque des questions au sujet de la localisation des maisons de saisonniers sont posées aux résidents permanents, les réponses sont vagues. Ces derniers ont connaissance de ce type d'habitat, mais ils ne semblent guère y porter une attention particulière.

Du côté des saisonniers eux-mêmes, leur disponibilité et leur souhait de parler demeurent restreints et rendent les entretiens complexes à mener. Pour autant, lors d'échanges plus ou moins formels avec ces derniers, il apparaît que ces lieux, peu visibles aux yeux des résidents et " masqués" par les employeurs, sont en réalité ceux d'un autre réseau de sociabilité. En effet, les saisonniers interrogés présentent dans un premier temps leur lieu de résidence, puis font assez rapidement référence à d'autres maisons ou appartements de saisonniers qu'ils connaissent et pratiquent à travers les relations qu'ils entretiennent avec leurs occupants. 
Les maisons des saisonniers présentent donc ainsi une double dimension : pour certains, ce sont des non-lieux, dont on connaît l'existence mais sans pouvoir (ou vouloir) les localiser, ce qui atténue leur qualité de lieu; pour d'autres, ceux qui les occupent, elles appartiennent au champ des lieux habités. Les saisonniers procurent ainsi à ces endroits une visée sociale à laquelle ils s'identifient. L'identification permet au non-lieu de devenir un lieu dans le sens où il est "chargé de valeurs communes dans lesquelles peuvent potentiellement se reconnaître des individus" (Lussault, in Lévy et Lussault (dir.), 2003 : 562), celles d'une condition de vie et d'une mobilité forte.

\section{Du non-lieu au lieu accaparé : les "campements" de saisonniers}

A la différence de l'exemple précédent, les « campements " de saisonniers sont des espaces que s'approprient les saisonniers pour établir leurs résidences. Ces «campements » correspondent à des regroupements de caravanes ou de camions aménagés, qui sont de tailles plus ou moins variables (figure 3). "Installer un campement " peut se traduire par l'accaparement d'un non-lieu par les saisonniers; qu'il s'agisse d'un parking, d'une bordure de forêt longeant une route, d'un terrain vague. Ces types d'endroits peuvent être considérés comme des non-lieux, des espaces de passage, sans portée sociale.

Ce type d'habitat est révélateur de la pénurie de logements pour les employés saisonniers au sein des stations de sports d'hiver. Les pouvoirs publics et les employeurs ont conscience de ce problème, mais ils se placent fréquemment dans une position de rejet de ce type d'habitat, considéré comme marginal et nuisible à l'image des stations de sports d'hiver haut de gamme. Les communes essaient donc soit de contrôler le développement de ces habitats mobiles regroupés en créant des zones réservées avec des contrôles effectués par la municipalité, soit de les interdire, repoussant ainsi la problématique dans des communes de fond de vallée dont le standing touristique n'est pas le même. La dynamique, visant à rendre invisibles les saisonniers au sein des espaces touristiques, correspond au principe de relégation, soit "l'expulsion d'une vie hors des espaces consacrés" (Le Blanc, 2009 : 14) ; les espaces consacrés étant ceux qui sont mis en lumière, mis en tourisme ${ }^{1}$. A la différence des lois contraignant les communes de plus de 5000 habitants à créer des aires d'accueil des gens du voyages (loi dite Besson du 5 juillet 2000), il n'existe aucune réglementation concernant les logements saisonniers, qu'ils soient ordinaires ou non (Bordigini, 2001).

\footnotetext{
${ }^{1}$ Les stratégies de relégation apparaissent être comme une des solutions apportées par les maires des communes de Megève, Val d'Isère et Saint-Martin-de-Belleville sur la question du logement des saisonniers, durant les entretiens réalisés en 2013.
} 
Figure 3 : Regroupement spontané d'habitats mobiles sur un terrain inoccupé (hameau du Fornet, Val d'Isère).

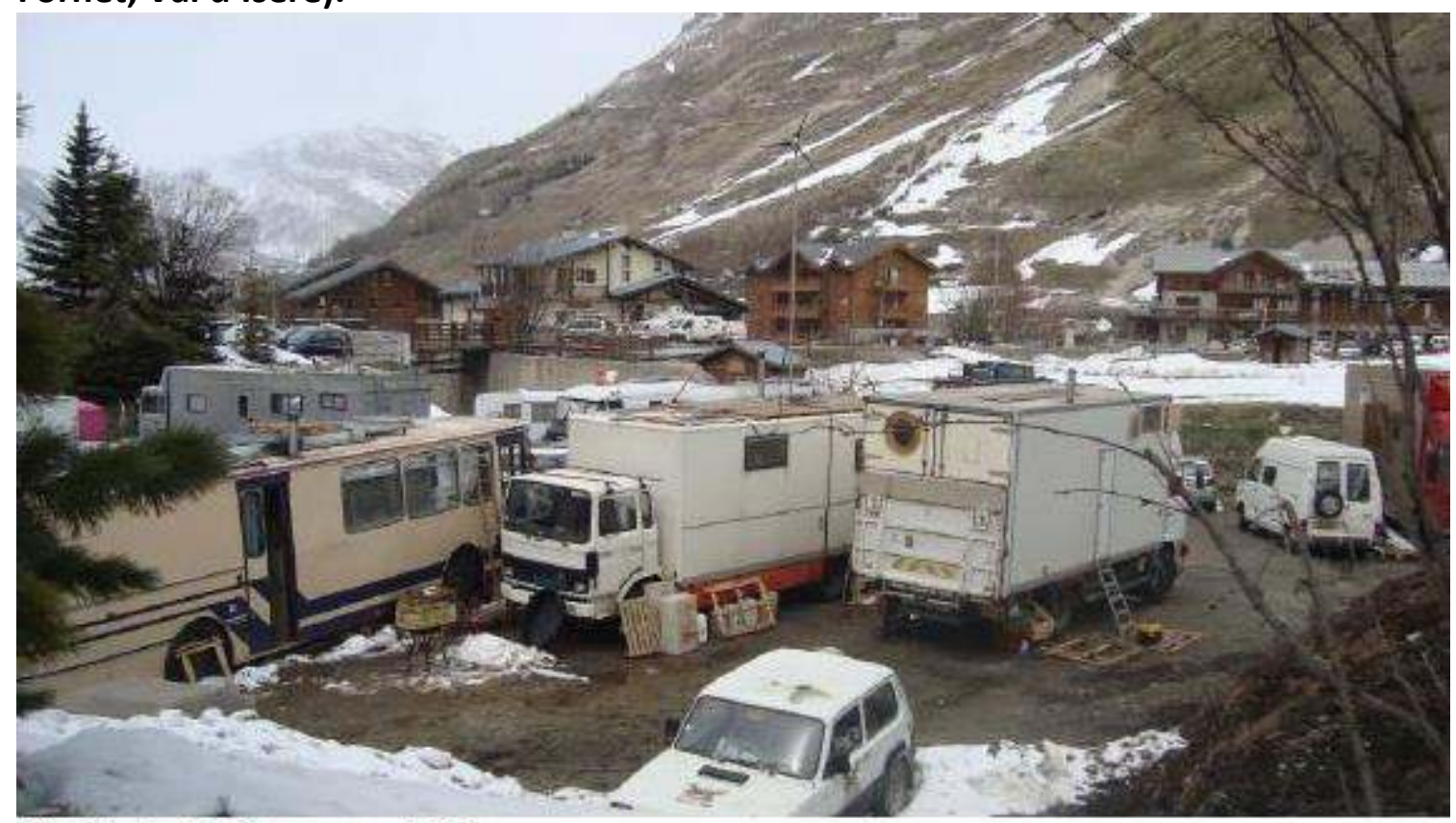

Cliché : S. Mollier, mars 2012.

L'appropriation de ces endroits par les saisonniers provoque leur changement de statut, transformant un non-lieu en lieu de vie à partir d'initiatives individuelles communes à un groupe. Le changement de statut spatial provoque un changement de statut social, qui se traduit par une relégation des saisonniers dans des espaces qui ont peu de considération pour les acteurs du tourisme, ce que M. Agier nomme "le lieu de la mise à l'écart » (2011 : 24), dans un contexte différent (les zones d'attente des demandeurs d'asile), mais avec des logiques d'exclusion similaires.

\section{Non-lieux versus espaces de l'entre-deux?}

Pour M. Augé, " un espace qui ne peut se définir ni comme identitaire, ni comme relationnel, ni comme historique définira un non-lieu " (1992: 100). Cette définition d'un anthropologue pose problème par la confusion entre espace et lieu, et par le phénomène d'appropriation de l'espace relevant plutôt du territoire. Ainsi, dans le cas des deux exemples précédemment présentés comme des non-lieux, ils s'avèrent être des lieux de vie, matérialisant des pratiques. L'auteur note également que «la possibilité du non-lieu n'est jamais absente de quelque lieu que ce soit " (id. : 134), mais l'inverse peut-il être possible ? Que cela soit dans le cadre des maisons de saisonniers ou celui des campements, les non-lieux se transforment en lieux aux yeux des occupants et également aux yeux des autorités gestionnaires. Nonlieux et lieux, plus que de s'enchevêtrer et de se mêler, sont des facettes d'un même espace, relevant de la pluralité des individus qui l'occupent. 
A travers une occupation qui est certes saisonnière, mais également répétitive, des non-lieux se transforment en lieux accaparés (les campements), en lieux de l'entre-deux (maisons de saisonniers) et en lieux appropriés lorsque l'occupation devient permanente (cas des saisonniers qui ont la possibilité de s'établir à l'année après l'obtention d'un poste fixe sur la station de sports d'hiver), et non plus comme des non-lieux, hormis pour les individus qui en nient l'existence, notamment dans le cas des campements. Cette modification de considération des lieux de passage en lieux de vie se produit grâce à une intensification de la perception, de la considération et de l'utilisation de ces derniers par les populations qui les occupent et les populations environnantes (figure 4).

Figure 4 : Du non-lieu au lieu approprié : la place des saisonniers dans les stations de sports d'hiver haut de gamme en France.

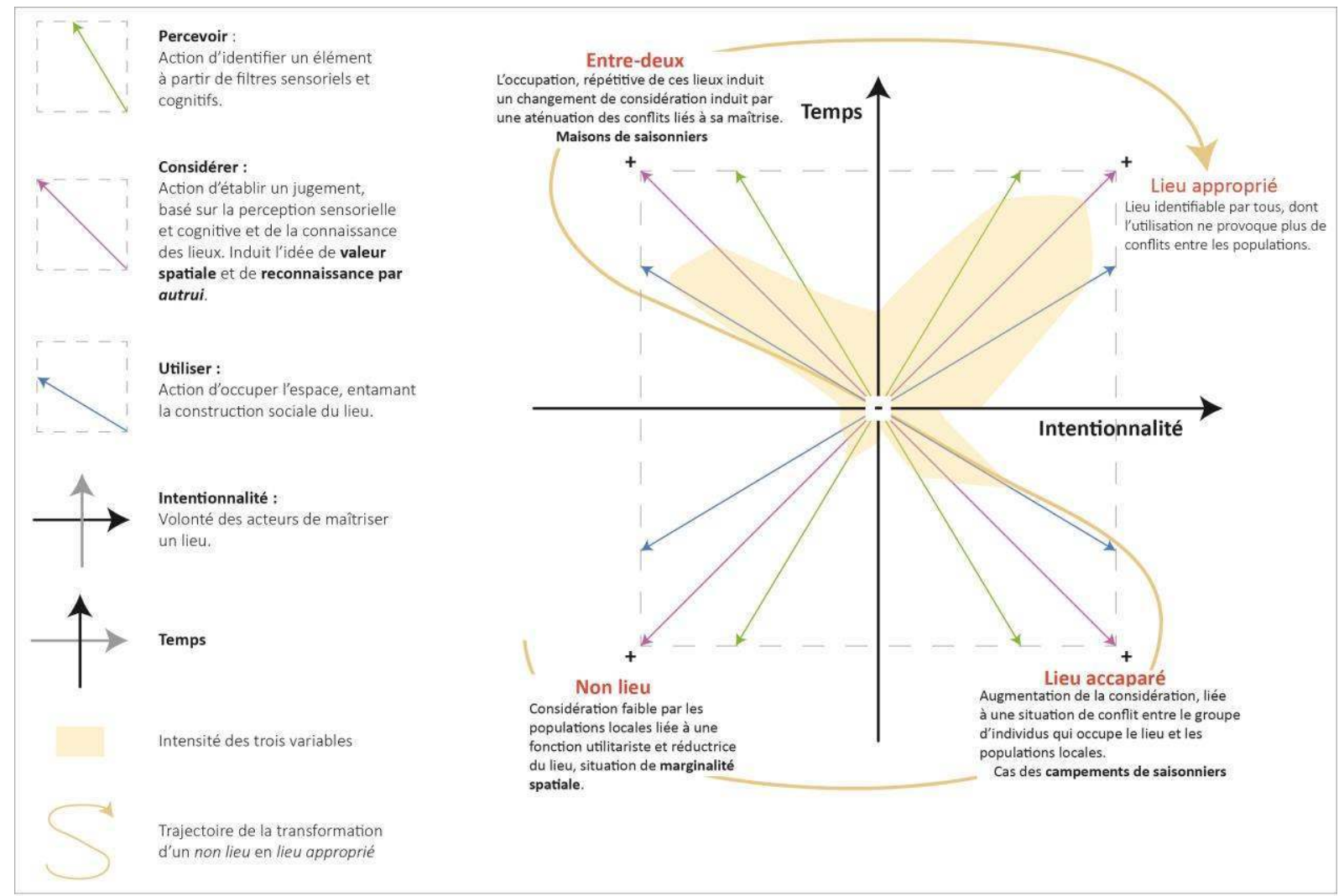

Conception et réalisation : L. Piquerey, décembre 2013.

La diversité des considérations à propos des lieux de vie des saisonniers et les formes d'occupations éphémères qu'ils représentent, les identifie comme des espaces de l'entredeux. A la fois espaces collectifs dans le mode de fonctionnement et espaces domestiques dans l'utilisation qu'en font les saisonniers, les lieux de vie des saisonniers sont des espaces en marge du système touristique qui ne peut fonctionner sans eux. Cette marginalité transparaît dans l'intérêt que leur portent les employeurs, ce type d'infrastructure étant principalement pensé dans une logique économique (liée à l'emploi), et également dans leur localisation, sur les franges de la station de sports d'hiver ou en périphérie proche de 
l'établissement, dans les espaces les moins attractifs (nuisances sonores, vues non dégagées...). Cette marginalisation des habitats non ordinaires est, comme le souligne A. Le Marchand, "dans la représentation du sens commun dominant, et dans l'administration, rejeté du côté de l'exclusion par la pauvreté » (2011 : 178).

\section{Conclusion}

Cette territorialisation des saisonniers au sein des stations de sports d'hiver haut de gamme résulte d'une volonté de mise à distance de ce type de population par les agents touristiques institutionnels. Plus que simplement physique, cette mise à distance renvoie à une certaine " invisibilité » de ces populations (impossibilité de localisation), comme par exemple en attribuant aux « campements » des terrains en marge, renforçant ainsi la marginalité sociale par une marginalité physique de ce type d'habitat. Par exemple, lors de nos entretiens, I'un des maires des stations de l'échantillon d'étude ayant dû faire face à la création spontanée d'un " campement » de saisonniers, l'exprimait ainsi : " pour régler le problème nous avons trouvé un terrain en contre bas de la route, caché par les arbres et en face de la police municipale. $»^{2}$

Cette citation illustre bien le caractère conflictuel entre les lieux de vie des saisonniers et le développement touristique des stations de sports d'hiver à travers une montée en gamme toujours plus significative. En effet, en dépit du fait que ces derniers soient partie prenante du système touristique, ils sont considérés comme des "impensés touristiques». Les espaces de vie des saisonniers se placent dans un espace de l'entre-deux, construit à travers le jeu du visible et du caché et de la mise à distance. Leur position marginale, la considération qui leur est portée tranche avec les lieux de la représentation touristique des stations de sports d'hiver haut de gamme, qui au contraire de ces derniers sont mis en avant et valorisés. La relégation à l'encontre des saisonniers vivant dans des habitats ordinaires s'accentue à l'égard de ceux qui résident dans les habitats mobiles, par rapport à la " norme d'habiter ».

L'invisibilité sociale des saisonniers, dans le sens où ils ne participent pas pleinement, du fait d'une faible reconnaissance par les acteurs locaux, à la vie publique de la commune, les place dans une situation d'entre-deux ; leur temps de présence saisonnier sur le territoire accentue cette difficulté à s'intégrer à la vie publique. Au demeurant, au-delà des stations de sports d'hiver haut de gamme françaises, des dynamiques de relégation identiques sont observées dans d'autres lieux de même type, en Suisse notamment (figure 5).

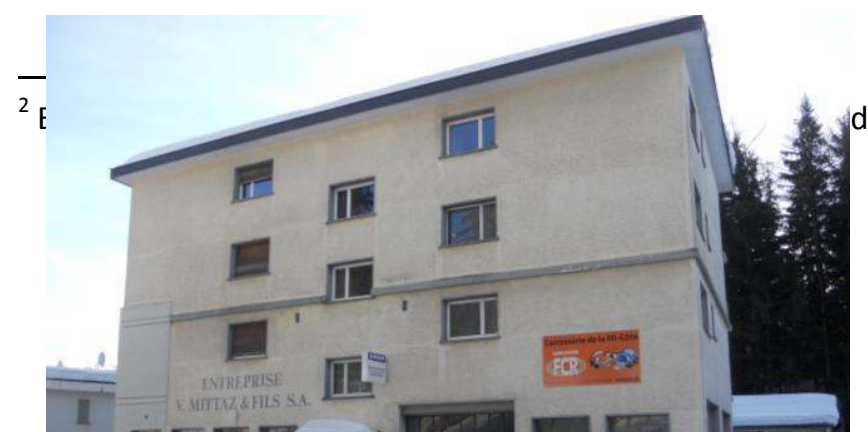

\section{Figure 5: Le Roc Neige}

Logement de saisonniers loué par l'Office du de Tourisme de Crans-Montana, situé audessus d'une carrosserie en activité six jours par semaine, et à l'intérieur d'une zone industrielle. Le bâtiment paraît vétuste, avec des problèmes d'isolation (fenêtres anciennes). L'office du Tourisme a connaissance de son état comme l'atteste 


\section{Bibliographie}

AGIER M., (2011) « L'encampement du monde », Plein droit, n90, pp. 21-24.

AUGE M., (1992), Non-Lieux. Introduction à une anthropologie de la surmodernité, Editions du Seuil, coll. la librairie du XXI ${ }^{\mathrm{e}}, 149 \mathrm{p}$.

AUGE M., (2010) "Retour sur les non-lieux. La transformation du paysage urbain ", Communication, $n^{\circ} 87$, pp. 171-178.

BORDIGINI M., (2001) «Terrain désigné, observation sous contrôle : quelques enjeux d'une ethnographie des Tsiganes », Ethnologie française, vol. 31, pp. 117-126.

BOUILLON F., (2009), Les Mondes du Squat, Editions des Presses Universitaires Françaises, coll. Partage et Savoir, 244p.

EQUIPE MIT, (2005), Tourisme 2. Moments de lieux, Editions Belin, 352p.

INSEE, 2010, données statistiques Rhône-Alpes.

LE BLANC G., (2009), L'invisibilité sociale, Editions des Presses Universitaires Françaises, coll. Pratiques théoriques, $197 p$.

LEVY J. \& LUSSAULT M. (dir.) (2003), Dictionnaire de la géographie et de l'espace des sociétés, Editions Belin, 1033p. 
LE MARCHAND A., (2011), Enclaves nomades. Habitat et travail mobile, Editions du croquant, $226 p$.

LEPRETRE G., (1996), L'épopée de Courchevel 1946-1996. 50 ans d'histoire de Courchevel racontée à ceux qui l'ont vécu, Editions La Fontaine de Siloé, 251p.

LUSSAULT M., (2009), De la lutte des classes à la lutte des places, Editions Grasset, 220p.

MOLLIER S., (2012), Les territoires du luxe en Savoie et en Haute-Savoie : étude géographique d'une visibilité de l'opulence à partir des cas de stations de ski (Megève, Courchevel, Val d'Isère) et de communes périurbaines (Veyrier-du-lac, Anthy-sur-Léman, Tresserve), Mémoire de master 1 en géographie, Université de Savoie, 81p.

PINCON M. et PINCON-CHARLOT M., (2005), Voyage en Grande Bourgeoisie, journal d'enquête, Editions Quadrige, coll. Essai, 186p.

PIQUEREY L., (2012), Entre fermeture et visibilité. La ségrégation et l'entre-soi dans les stations de sports d'hiver en Savoie et en Haute-Savoie, Mémoire de master 2 recherche en géographie, Université de Savoie, 109p

SOCQUET C., (1980), Megève et son passé, Editions France Couleur, 375p.

URIELY N., (2001) "Travelling workers and working tourists : variations accross the interaction between work and tourist ", International Journal of Tourism Research, $n^{\circ} 3, \mathrm{pp}$. 1-8. 